\title{
Ueber einige allgemeine Eigenschaften der Minimumsflächen.
}

(Von Herrn E. B. Christoffel in Zürich.)

Die sogenannten Minimumsflächen besitzen eine merkwürdige Eigenschaft, welche ich bei Gelegenheit meiner Abhandlung über die Bestimmung krummer Oberflächen durch locale Messungen auf denselben (dieses Journal Band 64) Herrn Weierstrass mitgetheilt habe. Dieselbe besteht in Folgendem.

1. Seien $S$ und $S^{\prime}$ zwei Minimumsflächen, d. h. solche, für welche die Summe der beiden Hauptkrümmungen in jedem Punkte $=0$ ist. Nachdem jede von diesen Flächen in eine beliebige Lage gebracht worden ist, wähle man auf ihnen Flächenstücke $T, T^{\prime}$ so aus, dass 1) auf keinem von beiden dieselbe Normalenrichtung zweimal, dagegen 2 ) jede auf dem einen stattinNormalenrichtung auch auf dem andern vorkommt.

Betrachtet man unter diesen Voraussetzungen einen Punkt $m^{\prime}$ von $T^{\prime}$ als Bild desjenigen Punktes $m$ von $T$, für welchen die gleiche Normalenrichṭng stattfindet, so wird $T^{\prime}$ ein in den kleinsten Theilen ähnliches Bild von $T$.

Bei diesem Satze ist der Fall nicht ausgeschlossen, dass die Flächen $S, S^{\prime}$ sich nur durch ihre Lage im Raume von einander unterscheiden. Durch blosse Drehung einer Minimumsfläche kann man also beliebig viele Abbildungen derselben auf sich selbst erhalten.

2. Der obige Satz bleibt bestehen, wenn eine der Flächen $S, S^{\prime}$ durch eine Kugel ersetzt wird.

Aus diesem zweiten Satze folgt der erste durch Wiederholung. Da ausserdem alle Abbildungen einer Kugelfläche auf einer Ebene bekannt sind, so sind es auch alle Abbildungen einer Minimumsfläche, wie zuerst von Herrn Weingarten gefunden worden ist *).

Ich habe mich veranlasst gesehen, diesen Gegenstand weiter zu verfolgen, und namentlich zu untersuchen, welche Flächen $T$ überhaupt die Eigenschaft haben, dass sich ihnen eine zweite $T^{\prime}$ zuordnen lässt, welche, ohne zu $T$ homothetisch zu sein, ein in den kleinsten Theilen ähnliches Bild von $\boldsymbol{T}$ liefert, wenn sie nach obigem Princip auf $T$ bezogen wird. Von dieser Unter-

*) Vergl. die reichhaltige Abhandlung: Ueber die Oberflächen, für welche einer der beiden Hauptkrümmungshalbmesser eine Function des andern ist. Dieses Journal Band 62, pag. 164, 165. 
suchung sind selbstverständlich alle diejenigen Flächen $T$ ausgeschlossen, auf denen jede überhaupt vorhandene Normalenrichtung eine Linie bestimmt, also die auf eine Ebene abwickelbaren Flächen.

Es hat sich herausgestellt, dass die verlangte Eigenschaft eine Flächenfamilie begründet, welche sich auch als das System derjenigen Flächen definiren lässt, die ein ebenes Bild gestatten, in welchem ihre Krümmungslinien durch zwei Schaaren zu einander senkrechter Geraden dargestellt werden. Es besitzt hiernach jede Fläche, an welcher die zuerst erwähnte Eigenschaft nachgewiesen ist, auch die zweite, und umgekehrt.

Diese Flächenfamilie ist sehr umfassend; mittelst der Formel B. des art. V., welche das Kriterium derselben enthält, findet man leicht, dass zu ihr nicht bloss die Minimumsflächen im gewöhnlichen Sinne, sondern allgemein diejenigen gehören, deren mittlere Krümmung constant ist, also die Minimumsflächen über gegebenem Volumen; ausserdem gehören hierhin alle Flächen zweiten Grades, alle Rotationsflächen, u. s. w.

In dieser Familie entspricht jeder Fläche $T$ eine andere $T^{\prime}$, und im Allgemeinen auch nur eine nebst ihren homothetischen, welche durch $T$ ihrer Gestalt und Lage nach bestimmt ist, wenn von Translationen ohne Drehungen abgesehen wird, die an der Abbildung nichts ändern. Die einzige Ausnahme hiervon bildet die Kugel, für welche die Aufgabe, $\boldsymbol{T}^{\prime}$ zu finden, unbestimmt ist, weil sie selbst jeder beliebigen Minimumsfläche entspricht.

Da die einfachen Hülfsmittel, welche ich bei dieser Untersuchung benutzt habe, sich auch bei anderen Gelegenheiten als brauchbar erweisen, so erlaube ich mir, im Folgenden zu zeigen, wie sich im Anschlusse an einen directen Beweis der obigen Sätze über Minimumsflächen die übrigen Resultate ergeben haben. Jene Sätze selbst habe ich ursprünglich aus dem art. IV. meiner oben erwähnten Arbeit erhalten.

\section{I.}

Wir beginnen unsere Untersuchung mit der Aufstellung der geometrischen Bedingungen, welche erforderlich sind, damit zwei Flächen $T, T^{\prime}$ einander ähnlich werden, wenn jedem Punkte $m$ von $T$ sein Bild $m^{\prime}$ auf $T^{\prime}$ durch die Bedingung gleicher Normalenrichtung zugeordnet wird, in welchem Falle.m und $m^{\prime}$ entsprechende Punkte heissen sollen.

$\mathrm{Zu}$ dem Zwecke bestimme man nach Gauss (Disqu. gen. circa superf. c. I.) eine Richtung im Raume mit Hülfe einer Kugel $K$ vom Halbmesser 1 durch den Punkt, in welchem ein gleichgerichteter Halbmesser auf der Kugel- 
fläche endigt. Sind also $N, N^{\prime}$ die Normalen der Flächen $T, T^{\prime}$ in den Punkten $m, m^{\prime}$, so werden diese Punkte einander entsprechen, sobald beide Normalen dem nämlichen Punkte $\mathfrak{R}$ von $\boldsymbol{K}$ entsprechen. Damit ferner zwei von entsprechenden Punkten $m, m^{\prime}$ ausgehende Linienelemente $\partial s, \partial s^{\prime} \operatorname{der}$ Flächen $T$; $T^{\prime}$ in entsprechenden Punkten endigen, müssen auch in ihren Endpunkten die Normalen dem nämlichen Punkte $\mathfrak{P}$ von $K$ entsprechen. Der unendlich kleine sphärische Bogen $\mathfrak{N P}=\Delta$ giebt dann den Winkel an, um.den sich die Normale $N$ dreht, wenn ihr Fusspunkt von $m$ aus $\partial s$ beschreibt.

Wir haben nun zu untersuchen, welche Bedingungen erforderlich sind, damit das Verhältniss der entsprechenden Linienelemente $\partial s, \partial s^{\prime}$ von der Lage des ihre Endpunkte bestimmenden Punktes $\mathfrak{B}$ unabhängig wird.

Seien $\partial N_{1}, \partial N_{2}$ in $m$ beginnende Bogenelemente der beiden Krümmungslinien von $T, \varrho_{1}, \varrho_{2}$ die ihnen entsprechenden Krümmungshalbmesser der Fläche. Ferner sei $\delta_{2}$ der Winkel, um den sich $\varrho_{1}$ dreht, wenn sein Fusspunkt $\partial N_{1}$ durchläuft, ebenso $\delta_{1}$ der Winkel, um den sich $\varrho_{2}$ dreht, wenn sein Fusspunkt $\partial N_{2}$ durchläuft, also abgesehen vom Zeichen $\partial N_{1}=\varrho_{1} \delta_{2}, \partial N_{2}=\varrho_{2} \delta_{1}$ und $\partial s^{2}=\varrho_{1}^{2} \delta_{2}^{2}+\varrho_{2}^{2} \delta_{1}^{2}$. Haben $\varrho_{1}^{\prime}, \varrho_{2}^{\prime}, \delta_{1}^{\prime}, \delta_{2}^{\prime}$ dieselbe Bedeutung für die Fläche $T^{\prime}$ im Punkte $m^{\prime}$, so wird $\partial s^{\prime 2}=\varrho_{1}^{\prime 2} \delta_{2}^{\prime 2}+\rho_{2}^{\prime 2} \delta_{1}^{\prime 2}$ und mithin das zu untersuchende Verhältniss

$$
\left(\frac{\partial s^{\prime}}{\partial s}\right)^{2}=\frac{\varrho_{1}^{\prime 2} \delta_{2}^{\prime 2}+\varrho_{2}^{\prime 2} \delta_{1}^{\prime 2}}{\varrho_{1}^{2} \delta_{2}^{2}+\varrho_{2}^{2} \delta_{1}^{2}} .
$$

Daraus ergeben sich die Sätze über die Minimumsflächen sofort. Nimmt man nämlich $\varrho_{1}^{\prime 2}=\varrho_{2}^{\prime 2}, \varrho_{1}^{2}=\varrho_{2}^{2}$, d. h. für $T$ und $T^{\prime}$ Minimums- oder Kugelflächen, so heben die Drehungswinkel sich weg, indem aus der Betrachtung der Kugel $\boldsymbol{K} \boldsymbol{A}^{2}=\delta_{1}^{2}+\delta_{2}^{2}=\delta_{1}^{\prime 2}+\delta_{2}^{\prime 2}$ folgt.

Ist nun, um zur allgemeinen Untersuchung überzugehen, $o$ das Centrum der Kugel $K$ und sind $\mathfrak{N}_{1}, \mathfrak{N}_{2}, \mathfrak{N}_{1}^{\prime}, \mathfrak{R}_{2}^{\prime}$ diejenigen Punkte des durch $\mathfrak{R}$ als Pol bestimmten Aequators, welche den Richtungen $\partial N_{1}, \partial N_{2}, \partial N_{1}^{\prime}, \partial N_{2}^{\prime}$ der Krümmungslinien von $T$ und $T^{\prime}$ entsprechen, so folgt, weil der Punkt $\mathfrak{P}$ von $K$ sowohl dem Endpunkte von $\partial s$, als auch dem von $\partial s^{\prime}$ entspricht, dass der Uebergang von $\mathfrak{N}$ nach $\mathfrak{P}$ auf folgende zwei Arten bewirkt werden kann:

1. indem man zwei unendlich kleine Drehungen um die Axen $o \Re_{1}$, $o \mathfrak{R}_{2}$ zusammensetzt, von denen die erste den Winkel $\delta_{1}$, die andere den Winkel $\delta_{2}$ beträgt;

2. indem man zwei die Winkel $\delta_{1}^{\prime}, \delta_{2}^{\prime}$ betragende Drehungen um die Axen $o \mathfrak{N}_{1}^{\prime}, o \mathfrak{R}_{2}^{\prime}$ zusammensetzt. 
Dies festgestellt, zählen wir auf dem Aequator von $\mathfrak{N}_{1}$ aus Längen, die nach $\mathfrak{R}_{2}$ hin wachsen und setzen fest, was offenbar gestatlet ist, dass von den Punkten $\mathfrak{R}_{1}^{\prime}, \mathfrak{N}_{2}^{\prime}$ der zweite die grössere Länge hat. Ist alsdann $\alpha$ die Länge von $\mathfrak{N}_{1}^{\prime}, \lambda$ die Länge des Meridians $\mathfrak{N}$, so wird, weil alle Bogen $\delta$ unendlich klein sind, $\delta_{1}=\Delta \sin \lambda, \delta_{2}=\Delta \cos \lambda, \delta_{1}^{\prime}=\Delta \sin (\lambda-\alpha), \delta_{2}^{\prime}=\Delta \cos (\lambda-\alpha)$, mithin

$$
\left(\frac{\partial s^{\prime}}{\partial s}\right)^{2}=\frac{\varrho_{1}^{\prime 2} \cos ^{2}(\lambda-\alpha)+\varrho_{2}^{\prime 2} \sin ^{2}(\lambda-\alpha)}{\varrho_{1}^{2} \cos \lambda^{2}+\varrho_{2}^{2} \sin \lambda^{2}},
$$

sobald $\partial s, \partial s^{\prime}$ in entsprechenden Punkten anfangen und endigen.

Dieser Ausdruck soll nun von der Lage des Punktes $\mathfrak{P}, \mathrm{d}$. h. von $\lambda$ unabhängig sein. Man erkennt ohne Mühe, dass diese Bedingung für jeden beliebigen Werth von $\alpha$, d. h. unabhängig von den Richtungen, in denen die Krümmungslinien der Flächen $T, T^{\prime}$ von $m, m^{\prime}$ ausgehen, nur dann erfüllt wird, wenn zugleich $\rho_{1}^{\prime 2}=\varrho_{2}^{\prime 2}, \varrho_{1}^{2}=\varrho_{2}^{2}$ ist, was den bereits oben erledigten Fall liefert.

Ist diese Bedingung nicht erfüllt, so kann das Verhältniss der beiden Linienelemente nur noch in dem Falle von $\lambda$ unabhängig sein, wenn $\alpha=0$ oder ein Vielfaches von $90^{\prime \prime}$ ist. In allen diesen Fällen wird jedem der beiden von $m$ ausgehenden Krümmungslinienelemente eines der beiden von $m^{\prime}$ ausgehenden parallel; wir schliessen daher keinen Fall aus, wenn wir

$$
\alpha=0
$$

- setzen, d. h. fordern, dass $\partial N_{1}^{\prime}$ mit $\partial N_{1}, \partial N_{2}^{\prime}$ mit $\partial N_{2}$ gleiche Richtung habe. Dann wird

$$
\left(\frac{\partial s^{\prime}}{\partial s}\right)^{2}=\frac{\varrho_{1}^{\prime 2} \cos \lambda^{2}+\varrho_{2}^{\prime 2} \sin \lambda^{2}}{\varrho_{1}^{2} \cos \lambda^{2}+\varrho_{2}^{2} \sin \lambda^{2}}
$$

und dies wird nur dann von $\lambda$ unabhängig, wenn $\varrho_{1}^{\prime 2}: \varrho_{1}^{2}=\varrho_{2}^{\prime 2}: \varrho_{2}^{2}$, d. h. entweder $\frac{\varrho_{1}^{\prime}}{\varrho_{1}}=\frac{\varrho_{2}^{\prime}}{\varrho_{2}}$, oder $\frac{\varrho_{1}^{\prime}}{\varrho_{1}}=-\frac{\varrho_{2}^{\prime}}{\varrho_{2}}$ wird.

Die zweite von diesen beiden Lösungen liefert den allgemeinen Satz:

Wenn zwei Flächen $T$ und $T^{\prime}$ in der Beziehung zu einander stehen, dass in je zwei durch gleiche Normalenrichtung einander entsprechenden Punkten $m, m^{\prime}$ derselben 1) auch die Hauptschnitte beider Flächen einander parallel werden, und 2) zwischen den Verhältnissen der Krümmungen paralleler Hauptschnitte die Relation

$$
\frac{\varrho_{1}^{\prime}}{\varrho_{1}}+\frac{\varrho_{2}^{\prime}}{\varrho_{2}}=0
$$

stattfindet, so wird $T^{\prime}$ ein in den kleinsten Theilen ähnliches Bild von $T$, wenn jeder Punkt $m^{\prime}$ als das Bild des entsprechenden Punktes $m$ angesehen wird. 
In diesem Satze sind die auf die Minimumsflächen bezüglichen ebenfalls enthalten. Nimmt man nämlich für $T^{\prime}$ eine Minimumsfläche, so folgt aus der zweiten Bedingung, dass $T$ eine Kugelfläche sein muss, und diese genügt offenbar auch der ersten Bedingung.

Die erste Lösung führt auf ganz elementare Resultate, die hauptsächlich als Vervollständigung des vorigen Satzes aufzufassen sind. In der That fordert dieselbe, wie leicht zu sehen ist, und auch aus den folgenden Untersuchungen hervorgeht, nichts anderes, als dass $T$ und $T^{\prime}$ homothetisch sind, woraus dann freilich, da die Aehnlichkeit für beliebig grosse perspectivisch liegende Theile vorhanden ist, auch die Aehnlichkeit der kleinsten Theile folgt.

Es ergiebt sich aber hieraus, dass ausser den durch den ersten Satz bestimmten Flächenpaaren und ihren homothetischen keine andern existiren, welche die von uns geforderten Eigenschaften besitzen.

II.

Es wirft sich jetzt die Frage auf, wie und unter welchen Voraussetzungen die Bedingungen des obigen Satzes befriedigt werden können.

Zu dem Zwecke bemerken wir zunächst, was aus der zweiten Bedingung folgt, dass in entsprechenden Punkten nothwendig die eine Fläche gewölbt, die andere sattelförmig gebogen sein muss. Um über bestimmte Vorstellungen zu verfügen, wollen wir daher, was auf den schliesslichen Verlauf der Untersuchung keinen Einfluss mehr haben wird, festsetzen, es seien $\varrho_{1}$, $\varrho_{2}, \varrho_{1}^{\prime}$ positiv, $\varrho_{2}^{\prime}$ dagegen sei negativ.

Wir bezeichnen nun die rechtwinkligen Coordinaten von $m$ durch $x, y, z$, durch $x^{\prime}, y^{\prime}, z^{\prime}$ diejenigen von $m^{\prime}$, ausserdem die Richtungscosinus der Normale der ersten und zweiten Krümmungslinie in $m$, also wegen des geforderten Parallelismus auch in $m^{\prime}$ durch $a, b, c ; a_{1}, b_{1}, c_{1} ; a_{2}, b_{2}, c_{2}$, wobei die Vorzeichen der Grössen $a, b, c$ so gewählt sein sollen, dass die durch sie bestimmte Richtung der Normale derjenigen entgegengesetzt wird, in welcher von der Oberfläche aus die positiven Krümmungshalbmesser aufgetragen werden.

In Folge des Parallelismus der Krümmungslinien werden auch die Drehungswinkel um die zu ihren Tangenten parallelen Axen für beide Flächen gleich, also $\delta_{1}^{\prime}=\delta_{1}, \delta_{2}^{\prime}=\delta_{2}$.

Wir bestimmen nun zunächst die Grössen, um welche die Coordinaten von $m$ und $m^{\prime}$ und die Richtnngscosinus der Normale in Folge dieser Drahungen zunehmen. 
Dreht man die Normale um eine zur Tangente der zweiten Krümmungslinie parallele und durch das Krümmungscentrum des ersten Hauptschnitts gehende Axe herum, bis der Drehungswinkel $\delta_{2}$ geworden ist, so durchläuft ihr Fusspunkt auf beiden Flächen die erste Krümmungslinie, und weil $\varrho_{1}, \varrho_{1}^{\prime}$ positiv sind, beide in derselben Richtung. Die zurückgelegten Wege sind $\partial N_{1}=\varrho_{1} \delta_{2}, \partial N_{1}^{\prime}=\varrho_{1}^{\prime} \delta_{2}$, mithin die Zunahmen von $x$ und $x^{\prime}$ :

$$
\partial x=a_{1} \varrho_{1} \delta_{2}, \quad \underset{1}{\partial} x^{\prime}=a_{1} \varrho_{1}^{\prime} \delta_{2},
$$

vorausgesetzt, dass man den Drehungswinkel $\delta_{2}$ positiv oder negativ nimmt, jenachdem $m$ sich in der Richtung $a_{1} b_{1} c_{1}$, oder ihr entgegen bewegt.

Nach der Lehre von der Krümmung der Flächen ist aber auch $\underset{1}{\partial x}=\varrho_{1} \partial a$, $\partial x^{\prime}=\varrho_{1}^{\prime} \partial a$, woraus in beiden Fällen

folgt.

$$
\partial a=a_{1} \delta_{2}
$$

Dreht man die Normale um eine zur Anfangsrichtung der ersten Krümmungslinie parallele und durch das Krümmungscentrum des zweiten Hauptschnittes gehende Axe herum, bis der Drehungswinkel $\delta_{1}$ geworden ist, so durchläuft ihr Fusspunkt auf beiden Flächen die zweite Krümmungslinie, aber in entgegengesetzten Richtungen. Sind $\partial N_{2}, \partial N_{2}^{\prime}$ die wirklich zurückgelegten Wege, so nimmt $x$ um $a_{2} \partial N_{2}$ zu, $x^{\prime}$ um $a_{2} \partial N_{2}^{\prime}$ ab. Nun ist $\partial N_{2}=\varrho_{2} \delta_{1}, \partial N_{2}^{\prime}=-\varrho_{2}^{\prime} \delta_{1}$, also wachsen $x$ und $x^{\prime}$ um

$$
\underset{2}{\partial x}=a_{2} \varrho_{2} \delta_{1}, \quad \underset{2}{\partial x^{\prime}=a_{2} \varrho_{2}^{\prime} \delta_{1},}
$$

wieder vorausgesetzt, dass $\delta_{1}$ positiv oder negativ genommen wird, jenachdem $m$ sich in der Richtung $a_{2} b_{2} c_{2}$ oder ihr entgegen bewegt.

Da ausserdem $\underset{2}{\partial x}=\varrho_{2} \partial a, \underset{2}{\partial x^{\prime}}=\underset{2}{\varrho_{2}^{\prime}} \partial a$ ist, so folgt

$$
\partial a=a_{2} \delta_{1} \text {. }
$$

Daraus ergeben sich für die vollständigen Differentiale die folgenden wichtigen Formeln:

$$
\begin{array}{lll}
\partial x=a_{1} \varrho_{1} \delta_{2}+a_{2} \varrho_{2} \delta_{1}, & \partial y=b_{1} \varrho_{1} \delta_{2}+b_{2} \varrho_{2} \delta_{1}, & \partial z=c_{1} \varrho_{1} \delta_{2}+c_{2} \varrho_{2} \delta_{1}, \\
\partial a=a_{1} \delta_{2}+a_{2} \delta_{1}, & \partial b=b_{1} \delta_{2}+b_{2} \delta_{1}, & \partial c=c_{1} \delta_{2}+c_{2} \delta_{1}, \\
\partial x^{\prime}=a_{1} \varrho_{1}^{\prime} \delta_{2}+a_{2} \varrho_{2}^{\prime} \delta_{1}, & \partial y^{\prime}=b_{1} \varrho_{1}^{\prime} \delta_{2}+b_{2} \varrho_{2}^{\prime} \delta_{1}, & \partial z^{\prime}=c_{1} \varrho_{1}^{\prime} \delta_{2}+c_{2} \varrho_{2}^{\prime} \delta_{1},
\end{array}
$$

von denen die auf die Fläche $T$ allein bezüglichen für jede beliebige Fläche gelten. Aus den bei ihrer Herleitung unterschiedenen Fällen ergiebt sich 
übrigens, dass diese Formeln von den zu Eingange über die Vorzeichen der Krümmungshalbmesser gemachten Voraussetzungen unabhängig, also auf beide Fälle des vorigen art. anwendbar sind.

III.

Setzt man nun, um die erste Lösung des art. I. zu befriedigen, $\varrho_{1}^{\prime}=k \varrho_{1}$, $\varrho_{2}^{\prime}=k \varrho_{2}$, so wird $\partial x^{\prime}=k \partial x, \partial y^{\prime}=k \partial y, \partial z^{\prime}=k \partial z$. Damit diese Gleichungen eine Fläche $T^{\prime}$ bestimmen können, müssen ihre rechten Seiten vollständige Differentiale sein. Betrachtet man $z$ als Function von $x, y$, so folgt aus der ersten Gleichung, dass $k$ von $y$, aus der zweiten, dass es von $x$ unabhängig, also constant sein muss. Diese Lösung liefert also, wie schon früher angewandt worden ist, zu jeder beliebigen Fläche ihre sämmtlichen homothetischen.

IV.

Zur Befriedigung der zweiten Lösung

$$
\frac{\varrho_{1}^{\prime}}{\varrho_{1}}+\frac{\varrho_{2}^{\prime}}{\varrho_{2}}=0
$$

setzen wir $\varrho_{2}^{\prime}=H \varphi_{2}, \varrho_{1}^{\prime}=-H \varrho_{1}$. Dann wird $\underset{1}{\partial x^{\prime}}=-H \underset{1}{\partial x}, \underset{2}{\partial x^{\prime}=H \partial x}{ }_{2}$, u. s. w.

Nennt man nun diejenige Seite einer Fläche, von welcher ihre Normalen ausgehen, ihre Vorderseite, so ergiebt sich aus diesen Formeln zunächst, dass im vorliegenden Falle die Vorderseite von $T$ auf der Rückseite von $T^{\prime}$ abgebildet wird, und ungekehrt, vorausgesetzt, dass man unter einer Abbildung eine im gewöhnlichen Sinne, also nicht verkehrt ähnliche versteht.

Sodann ergeben sich für die vollständigen Differentiale die Gleichungen

$$
\partial x^{\prime}=\boldsymbol{H}(\underset{2}{\partial x}-\underset{1}{\partial x}), \quad \partial y^{\prime}=\boldsymbol{H}(\underset{2}{\partial y}-\partial y), \quad \partial \boldsymbol{z}^{\prime}=\boldsymbol{H}(\underset{2}{\partial z}-\underset{1}{\partial z}),
$$

von denen zunächst gezeigt werden soll, dass sie aus den Bedingungen unserer Untersuchung nicht bloss folgen, sondern dieselben auch umgekehrt nach sich ziehen.

Sind nämlich $a, b, c$ die Richtungscosinus der Normale von $T$, so sind sie es auch für $T^{\prime}$, weil aus den vorstehenden Gleichungen

$$
a \partial x^{\prime}+b \partial y^{\prime}+c \partial z^{\prime}=0
$$

folgt.

Da ferner die Krümmungslinienelemente $\partial N_{1}, \partial N_{2}$ auf einander senkrecht stehen, so folgt $\partial s^{\prime 2}=H^{2}\left(\partial N_{1}^{2}+\partial N_{2}^{2}\right)=H^{2} \partial s^{2}$, also ist $T^{\prime}$ ein in den kleinsten Theilen ähnliches Bild von $T$. 
Auf Grund dieser beiden Folgerungen ergiebt sich endlich nach art. I. auch der Parallelismus der Krümmungslinien beider Flächen.

Folglich enthalten die vorstehenden Differentialgleichungen alle für die gegenwärtige Aufgabe nothwendigen und ausreichenden Bedingungen. die Form

Setzt man $\frac{\varrho_{1} \varrho_{2}}{\varrho_{1}-\varrho_{2}} H=\Omega$, so lassen die obigen Gleichungen sich in

$$
\left\{\begin{array}{l}
\partial x^{\prime}=\Omega\left[2 \partial a-\left(\frac{1}{\varrho_{1}}+\frac{1}{\varrho_{2}}\right) \partial x\right] \\
\partial y^{\prime}=\Omega\left[2 \partial b-\left(\frac{1}{\varrho_{1}}+\frac{1}{\varrho_{2}}\right) \partial y\right] \\
\partial z^{\prime}=\Omega\left[2 \partial c-\left(\frac{1}{\varrho_{1}}+\frac{1}{\varrho_{2}}\right) \partial z\right]
\end{array}\right.
$$

bringen. Dieselbe zeigt sofort, dass unsere ursprüngliche Aufgabe, die Bedingungen zu ermitteln, damit zu einer gegebenen Fläche $T$ eine sie in der verlangten Weise abbildende existire, auf die Herstellung und Deutung der Integrabilitätsbedingungen zurückgeführt ist, welche erforderlich sind und hinreichen, damit die drei vorstehenden Ausdrücke zugleich vollständige Differentiale werden.

Es ist indessen zweckmässig, für diese Untersuchung die Differentialgleichungen so viel wie möglich in der ursprünglichen einfachsten Form beizubehalten.

\section{V.}

Wir setzen voraus, dass $x, y$ und $z$ als Functionen zweier von einander unabhängiger Variabeln $u_{1}, u_{2}$ in der Weise dargestellt sind, dass der Gleichung $\partial u_{1}=0$ diejenige Schaar Krümmungslinien von $T$ entspricht, welcher die vorhin als erste bezeichnete angehört, der Gleichung $\partial u_{2}=0$ die andere Schaar. Bezeichnet man daher die Derivirten von $x, y, z$ nach $u_{1}, u_{2}$ durch $x_{1}, x_{2}, x_{12}$, u. s. w., so wird

$$
\partial x=x_{2} \partial u_{2}, \quad \underset{2}{\partial x}=x_{1} \partial u_{1}, \quad \text { u. s. w. }
$$

Ferner folgt aus den Gleichungen $\underset{1}{\partial x}=\varrho_{1} \partial a, \underset{1}{\partial x}=\varrho_{2} \partial a$, wenn

gesetzt wird,

$$
\frac{1}{\varrho_{1}}=r_{1}, \quad \frac{1}{\varrho_{2}}=r_{2}
$$

$$
\frac{\partial a}{\partial u_{2}}=r_{1} x_{2}, \quad \frac{\partial a}{\partial u_{1}}=r_{2} x_{1}
$$


mithin durch Elimination von $a$

$$
\text { (1.) } \quad x_{12}=\frac{1}{r_{1}-r_{2}}\left(\frac{\partial r_{2}}{\partial u_{2}} x_{1}-\frac{\partial r_{1}}{\partial u_{1}} x_{2}\right)
$$

wozu noch zwei ähnliche Gleichungen für $y$ und $z$ kommen. Ausserdem ist

(2.) $\quad x_{1} x_{2}+y_{1} y_{2}+z_{1} z_{2}=0$.

Dies vorausgeschickt, nehmen die Differentialgleichungen des vorigen art. die folgende Form an:

$$
\left\{\begin{array}{l}
\partial x^{\prime}=H\left(x_{2} \partial u_{2}-x_{1} \partial u_{1}\right) \\
\partial y^{\prime}=H\left(y_{2} \partial u_{2}-y_{1} \partial u_{1}\right) \\
\partial z^{\prime}=H\left(z_{2} \partial u_{2}-z_{1} \partial u_{1}\right)
\end{array}\right.
$$

Für $\log H=Z$ liefert die erste als Integrabilitätsbedingung

oder wegen (1.)

$$
\frac{\partial Z}{\partial u_{1}} x_{2}+\frac{\partial Z}{\partial u_{2}} x_{1}+2 x_{12}=0
$$

$$
\left[\frac{\partial Z}{\partial u_{1}}-\frac{2}{r_{1}-r_{2}} \frac{\partial r_{1}}{\partial u_{1}}\right] x_{2}+\left[\frac{\partial Z}{\partial u_{2}}+\frac{2}{r_{1}-r_{2}} \frac{\partial r_{2}}{\partial u_{2}}\right] x_{1}=0
$$

Mit Rücksicht auf (2.) folgt aus dieser und den beiden hierzugehörigen Gleichungen, dass nothwendig

$$
\frac{\partial \boldsymbol{Z}_{1}}{\partial u_{1}}=\frac{\boldsymbol{Z}}{r_{1}-r_{2}} \frac{\partial r_{1}}{\partial u_{1}}, \quad \frac{\partial \boldsymbol{Z}_{2}}{\partial u_{2}}=-\frac{2}{r_{1}-r_{2}} \frac{\partial r_{2}}{\partial u_{2}}
$$

sein muss, und umgekehrt sind mit diesen beiden zugleich alle Integrabilitätsbedingungen erfüllt.

Damit aber diese beiden Gleichungen mit einander bestehen können, ist erforderlich und hinreichend, dass

$$
\text { (B.) } \frac{\partial}{\partial u_{2}}\left(\frac{1}{r_{1}-r_{2}} \frac{\partial r_{1}}{\partial u_{1}}\right)+\frac{\partial}{\partial u_{1}}\left(\frac{1}{r_{1}-r_{2}} \frac{\partial r_{2}}{\partial u_{2}}\right)=0
$$

sei. Ist diese Bedingung erfüllt, so liefern also die vorstehenden Ausdrücke für $\partial Z$ ein vollständiges Differential, und die so bestimmte Function

$$
\boldsymbol{H}=\boldsymbol{e}^{\boldsymbol{Z}}
$$

macht auch die Ausdrücke für $\partial x^{\prime}, \partial y^{\prime}, \partial z^{\prime}$ zu vollständigen Differentialen. Zugleich ist ersichtlich, dass der letztern Forderung auf keine andere Weise genügt werden kann.

Es bedarf nur noch der geometrischen Interpretation von (B.). Sei

$$
x_{1}^{2}+y_{1}^{2}+z_{1}^{2}=\xi_{1}^{2}, \quad x_{2}^{2}+y_{2}^{2}+z_{2}^{2}=\xi_{2}^{2},
$$

so folgt aus (1.) und (2.) 
(3.)

$$
\frac{1}{r_{1}-r_{2}} \frac{\partial r_{2}}{\partial u_{2}}=\frac{\partial \log \xi_{1}}{\partial u_{2}}, \quad \frac{1}{r_{1}-r_{2}} \frac{\partial r_{1}}{\partial u_{1}}=-\frac{\partial \log \xi_{2}}{\partial u_{1}}
$$

wodurch $(B$. $)$ in

$$
\frac{\partial^{2} \log \frac{\xi_{1}}{\xi_{2}}}{\partial u_{1} \partial u_{2}}=\mathbf{0}
$$

übergeht. Diese Gleichung fordert also, dass $\log \frac{\xi_{1}}{\xi_{2}}$ sich als Differenz einer bloss von $u_{1}$ und einer bloss von $u_{2}$ abhängigen Function darstellen lasse, dass also

$$
\frac{\xi_{1}}{\xi_{2}}=\frac{\psi\left(u_{1}\right)}{\psi\left(u_{2}\right)}
$$

dem Quotienten aus zwei solchen Functionen gleich sei.

Führt man nun, was an den frühern Voraussetzungen nichts ändert, mittelst der Gleichungen $\psi\left(u_{1}\right) \hat{\partial} u_{1}=\partial u_{1}^{\prime}, \psi\left(u_{2}\right) \hat{o} u_{2}=\partial u_{2}^{\prime}$ zwei neue Variabeln $u_{1}^{\prime}, u_{2}^{\prime}$ ein, so folgt, dass die Allgemeinheit unseres Resultates nicht geändert wird, wenn wir $\psi\left(u_{1}\right)$ und $\psi\left(u_{2}\right)$ constant und einander gleich setzen. Dann wird die Lösung von $(B$.)

$$
\text { (4.) } \dot{\xi}_{1}=\xi_{2} \text {. }
$$

Damit also die Gleichungen $(\boldsymbol{A}$.) durch passende Wahl von $\boldsymbol{H}$ integrabel gemacht werden können, ist erforderlich und hinreichend, dass das Quadrat des Linienelementes von $T$ sich in die Form $\partial s^{2}=\xi_{1}^{2}\left(\partial u_{1}^{2}+\partial u_{2}^{2}\right)$ bringen lasse, während $\partial u_{1}=0, \partial u_{2}=0$ die Differentialgleichungen der beiden Schaaren Krümmungslinien von $T$ sind. Wir haben hiernach den Satz:

Damit einer Fläche $T$ eine zweite $T^{\prime}$ in der oben verlangten Weise zugeordnet werden könne, ist erforderlich und hinreichend, dass $T$ ein in den kleinsten Theilen ähnliches ebenes Bild gestatte, in welchem seine Krümmungslinien durch zwei Schaaren zu einander senkrechter Geraden dargestellt werden.

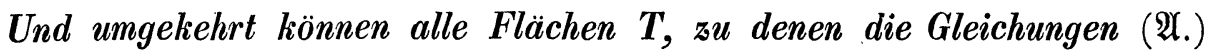
oder (A.) eine zugeordnete $T^{\prime}$ liefern, in dieser Weise auf einer Ebene abgebildet werden.

Bezeichnet man bei der Abbildung von $T$ auf der Ebene der $u_{1}, u_{2}$ das Vergrösserungsverhältniss durch $\frac{1}{m}$, so wird

$$
\partial s^{2}=m^{2}\left(\partial u_{1}^{2}+\partial u_{2}^{2}\right),
$$

und es ergeben sich für die Familie der Flächen $T$ aus (4.), (2.), (1.) und (3.) die Differèntialgleichungen : 


$$
\begin{gathered}
x_{1}^{2}+y_{1}^{2}+z_{1}^{2}=m^{2}, \quad x_{1} x_{2}+y_{1} y_{2}+z_{1} z_{2}=0, \quad x^{2}+y_{2}^{2}+z_{2}^{2}=m^{2}, \\
x_{12}=\frac{\partial \log m}{\partial u_{1}} x_{2}+\frac{\partial \log m}{\partial u_{2}} x_{1}, \\
y_{12}=\frac{\partial \log m}{\partial u_{1}} y_{2}+\frac{\partial \log m}{\partial u_{2}} y_{1}, \\
z_{12}=\frac{\partial \log m}{\partial u_{1}} z_{2}+\frac{\partial \log m}{\partial u_{2}} z_{1} .
\end{gathered}
$$

Ist $\boldsymbol{T}$ diesen Gleichungen gemäss bestimmt, so wird

$$
\partial \mathbf{Z}=-\mathbf{2} \frac{\partial \log m}{\partial u_{1}} \partial u_{1}-2 \frac{\partial \log m}{\partial u_{2}} \partial u_{2}
$$

also $Z=$ const. $-2 \log m$,

$$
\boldsymbol{H}=\frac{\boldsymbol{A}}{m^{2}},
$$

so dass man zur Bestimmung von $\boldsymbol{T}^{\prime}$ die integrabeln Gleichungen

$$
\begin{aligned}
& \partial x^{\prime}=\frac{A}{m^{2}}\left(x_{1} \partial u_{1}-x_{2} \partial u_{2}\right), \\
& \partial y^{\prime}=\frac{A}{m^{2}}\left(y_{1} \partial u_{1}-y_{2} \partial u_{2}\right) \\
& \partial z^{\prime}=\frac{A}{m^{2}}\left(z_{1} \partial u_{1}-z_{2} \partial u_{2}\right)
\end{aligned}
$$

erhält.

Es ist hier nicht der Ort, diesen Gegenstand durch Ausführung von Beispielen weiter zu verfolgen.

Zürich, 2. April 1867. 